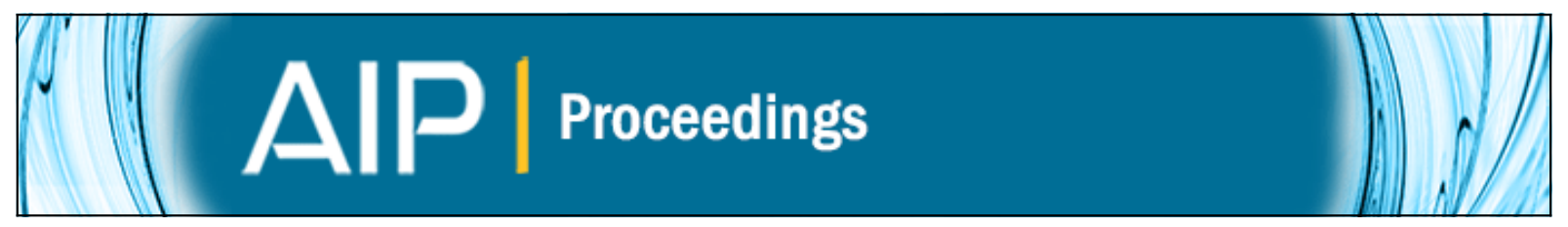

The first supernovae: Source density and observability of pair instability supernovae Jacob A. Hummel, Andreas H. Pawlik, Miloš Milosavljević, and Volker Bromm

Citation: AIP Conference Proceedings 1480, 376 (2012); doi: 10.1063/1.4754392

View online: http://dx.doi.org/10.1063/1.4754392

View Table of Contents: http://scitation.aip.org/content/aip/proceeding/aipcp/1480?ver=pdfcov

Published by the AIP Publishing

Articles you may be interested in

Pair-instability and super-luminous supernova discoveries at $z=2.05, z=2.50$, and $z=3.90$

AIP Conf. Proc. 1480, 200 (2012); 10.1063/1.4754355

The Observational Signatures of Primordial Pair-Instability Supernovae

AIP Conf. Proc. 1279, 116 (2010); 10.1063/1.3509246

Can Very Massive Stars Avoid Pair-Instability Supernovae?

AIP Conf. Proc. 990, 220 (2008); 10.1063/1.2905547

Possible Detection of a Pair Instability Supernova in the Modern Universe, and Implications for the First Stars AIP Conf. Proc. 990, 122 (2008); 10.1063/1.2905517

Optical observations of Type II supernovae

AIP Conf. Proc. 522, 123 (2000); 10.1063/1.1291705 


\title{
The First Supernovae: Source Density and Observability of Pair Instability Supernovae
}

\author{
Jacob A. Hummel, Andreas H. Pawlik, Miloš Milosavljević and Volker \\ Bromm
}

jhummel@astro.as.utexas.edu

\begin{abstract}
Theoretical models predict that some of the first stars ended their lives as extremely energetic Pair Instability Supernovae (PISNe). With energies approaching $10^{53} \mathrm{erg}$, these supernovae are expected to be within the detection limits of the upcoming James Webb Space Telescope (JWST) allowing observational constraints to be placed on the properties of the first stars. We estimate the source density of PISNe using a semi-analytic Press-Schecter based approach informed by cosmological simulations, with an upper limit of $\sim 0.2$ PISNe visible per JWST field of view at any given time. We find that the main obstacle to observing PISNe is their scarcity rather than their faintness. Given this we suggest a mosaic style search strategy for detecting PISNe from the first stars.
\end{abstract}

Keywords: cosmology: theory — dark ages, reionization, first stars — supernovae: general PACS: 98.80.Es, 97.60.Bw, 97.10.Bt

\section{INTRODUCTION}

The launch of the James Webb Space Telescope (JWST) will allow us to probe the epoch of the first stars in unprecedented detail. These Population III (Pop III) stars, which formed in $10^{5}-10^{6} M_{\odot}$ dark matter 'minihalos' at high redshifts were initially believed to be predominantly very massive. More recently, significant fragmentation has been found to occur in collapsing primordial gas, lowering the characteristic mass of the first stars to nearer $50 M_{\odot}$ and broadening the initial mass function [1]. The most massive of these stars may have died as pair-instability supernovae (PISNe), which should be within the detection limits of the JWST [2]. Our work complements previous investigations of the PISN rate (e.g., $[3,4,5])$ by including an investigation of the effects of stellar feedback on the PISN rate, considering Lyman-Werner (LW) feedback and chemical feedback. We describe here our model for the PISN rate and assess the ability of the JWST to detect PISNe from the first stars. For further details see [6], on which this work is based.

\section{METHODS}

After the first massive star forms in a minihalo, the ionizing radiation produced effectively halts further star formation until its death. The energy released by the first PISN disperses the gas in the halo, delaying subsequent star formation until the gas is able to recondense into more massive halos, which will likely be enriched beyond the critical metallicity for Pop II star formation. While it is possible that the first stars formed in small multiples [1], the number per minihalo is still of order unity, so we assume exactly 

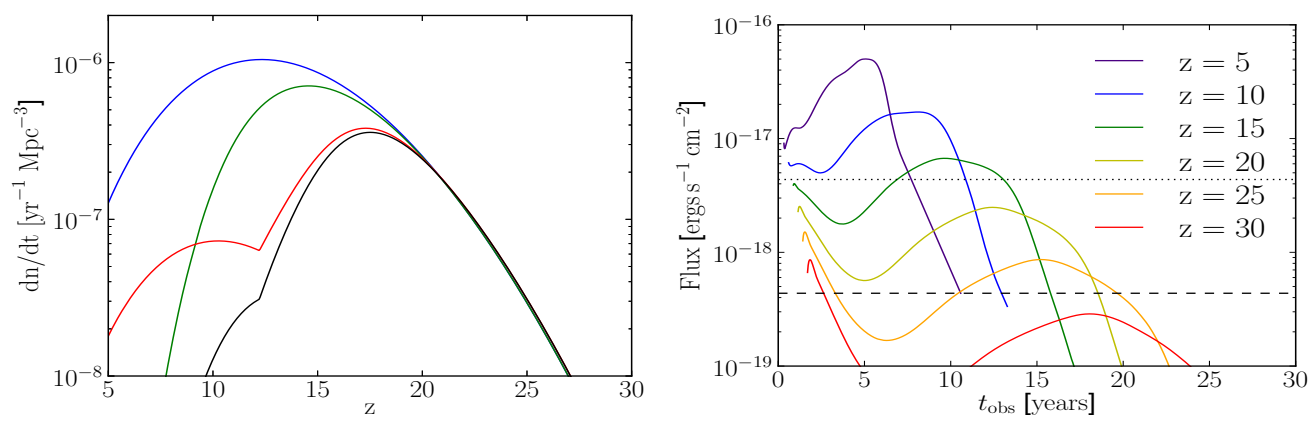

FIGURE 1. Left: The PISN rate in the upper limit of no feedback (blue), with chemical feedback (green), LW feedback (red) and the final predicted PISN rate (black). Right: Lightcurves for the our PISN model as it would be observed by JWST's F444W NIRCam filter at $z=5,10,15,20,25$ and 30. The flux limits for a $10^{6} \mathrm{~s}$ (dashed line) and $10^{4} \mathrm{~s}$ (dotted line) are shown for reference.

one PISN per minihalo, forming as soon as the halo exceeds the critical mass required for star formation. We then use the analytic Press-Schechter (PS) formalism for structure formation [7] to calculate the number density of critical mass minihalos at redshift $z$, estimating their formation rate $\dot{n}_{+}(z)$ using the expression derived by [8]. The resulting rate can be seen in the left panel of Figure 1 (blue).

Stellar feedback will suppress Pop III star formation in some minihalos and completely halt it in others, reducing the PISN rate. To asses the impact of Lyman-Werner (LW) feedback we employ a set of two cosmological simulations. The first simulation we employ is similar to simulation Z4 presented in [9], and does not include LW feedback. The second simulation differs only in the inclusion of LW feedback. The LW modulate PISN rate is shown in left panel of Figure 1 (red); note that once the critical mass reaches the atomic cooling threshold at $z \sim 12$, LW feedback is no longer efficient.

Gas that has been enriched beyond a critical metallicity of $Z_{\text {crit }} \sim 10^{-4} Z_{\odot}$ will no longer form Pop III stars. To estimate the impact of chemical feedback on the PISN rate, we use the results of [10], who estimate the probability that the gas in a newly formed halo is pristine. The resulting PISN rate is show in the left panel of Figure 1 (green) along with the final predicted PISN rate (black).

To estimate the observability of PISNe at high redshifts we consider the $250 M_{\odot}$ PISN model presented in [11]. Given the large mass ejected, the ejecta will remain optically thick until late times, so we make the reasonable assumption that the PISN emits as a blackbody for the majority of its visible lifetime. The resulting lightcurves as they would appear at various redshifts are shown in the right panel of Figure 1.

\section{DISCUSSION AND CONCLUSIONS}

The limiting factor in detecting PISNe will be the scarcity of sources rather than their faintness. PISNe should be readily detectable out to $z \sim 20$, but beyond a moderate exposure time their observability is controlled almost completely by the source densityapproximately one PISN per 5 JWST fields of view (FoVs) above $z=10$. This suggest that a mosaic style search strategy covering a large area with relatively short exposures 


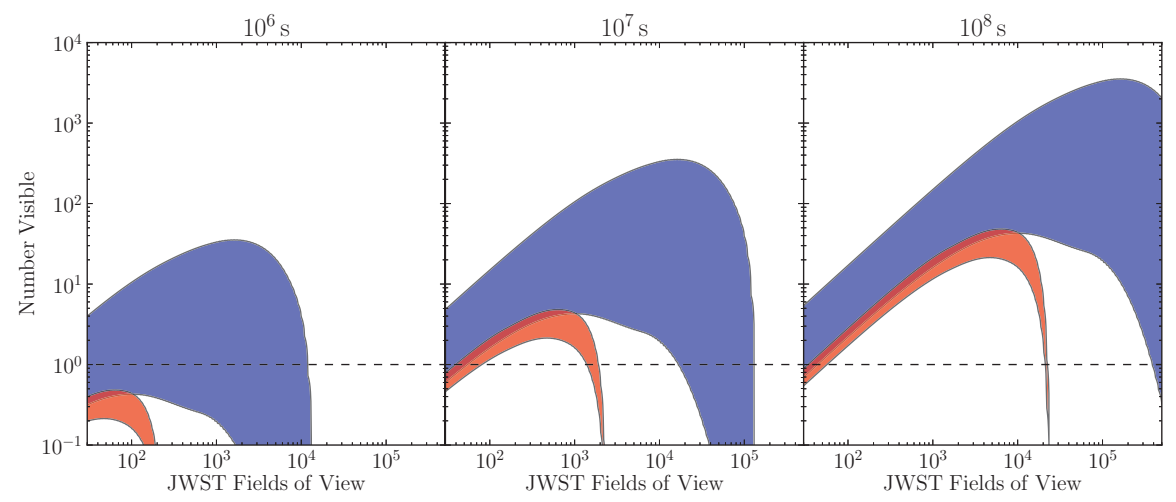

FIGURE 2. The total number of PISNe observable with a campaign of $10^{6}, 10^{7}$ and $10^{8} \mathrm{~s}$ (from left to right) as a function of survey area for our PISN model. In each case, the total campaign time is apportioned equally over the total survey area to determine the exposure time for individual pointings. The blue region represents all PISNe, the red only PISNe from $z>15$. Upper boundaries correspond to the no-feedback upper limit to the PISN rate and lower boundaries to the combined feedback rate.

will provide the highest likelihood of success. This is clear from Figure 2, where we have shown the number of PISNe detectable with the JWST as a function of survey area for campaigns totalling $10^{6}, 10^{7}$, and $10^{8} \mathrm{~s}$. While the detection of a PISN from a 'first' star at very high redshifts is possible given the detection limits of the JWST, the scarcity of sources at these redshifts means that such a detection would be contingent on serendipity. However, the detection of a PISN at lower redshifts appears to be within the realm of possibility.

\section{ACKNOWLEDGMENTS}

V.B. and M.M. acknowledge support from NSF grants AST-0708795 and AST-1009928 and NASA ATFP grant NNX09AJ33G. V.B. thanks the Max-Planck-Institut für Astrophysik for its hospitality during part of the work on this paper. The simulations were carried out at the Texas Advanced Computing Center (TACC).

\section{REFERENCES}

1. T. H. Greif, V. Springel, S. D. M. White, S. C. O. Glover, P. C. Clark, R. J. Smith, R. S. Klessen, and V. Bromm, ApJ 737, 75-92 (2011).

2. E. Scannapieco, P. Madau, S. E. Woosley, A. Heger, and A. Ferrara, ApJ 633, 1031-1041 (2005).

3. S. M. Weinmann, and S. J. Lilly, ApJ 624, 526-531 (2005).

4. J. H. Wise, and T. Abel, ApJ 629, 615-624 (2005).

5. M. Trenti, M. Stiavelli, and J. Michael Shull, ApJ 700, 1672-1679 (2009).

6. J. A. Hummel, A. H. Pawlik, M. Milosavljević, and V. Bromm, in press (arXiv:1112.5207v2), (2012).

7. W. H. Press, and P. Schechter, ApJ 187, 425-438 (1974).

8. S. Sasaki, PASJ 46, 427-430 (1994).

9. A. H. Pawlik, M. Milosavljević, and V. Bromm, ApJ 731, 54-71 (2011).

10. S. R. Furlanetto, and A. Loeb, ApJ 634, 1-13 (2005).

11. D. Kasen, S. E. Woosley, and A. Heger, ApJ 734, 102-115 (2011). 